\title{
SIMULAÇÃO DO RESFRIAMENTO DE BARRAS ENTRE O FORNO DE AQUECIMENTO E O LAMINADOR *
}

\author{
Kássio Nogueira Cançado ${ }^{1}$ \\ Lis Nunes Soares ${ }^{2}$ \\ Ricardo Junqueira Silva ${ }^{3}$ \\ Luis Fernando Oliveira Rodrigues ${ }^{4}$
}

\section{Resumo}

A perda de temperatura das barras de aço no intervalo entre a saída do forno e a entrada do laminador é fator determinante no processo de laminação e na qualidade do tubo produzido. $O$ estudo feito tem como objetivo modelar matematicamente 0 resfriamento destas barras durante o transporte entre o forno e o laminador, permitindo avaliar a temperatura mínima necessária para desenfornamento e também o máximo tempo de transporte sem comprometer o processo de laminação, possibilitando um melhor setup dos equipamentos reduzindo os custos de produção. Os resultados obtidos com o modelo matemático de resfriamento foram comparados com valores reais de temperatura coletados através de campanhas de medições com termopares instalados em uma barra e com medições de pirômetro. Valores de temperatura foram coletados com termopares em barras de 270, 230 e $180 \mathrm{~mm}$ de diâmetro. As medições com pirômetros foram feitas em barras de $180 \mathrm{~mm}$ de diâmetro. $\mathrm{O}$ modelo de resfriamento apresentou para todos os casos erro relativo às medições inferiores a 3\%.

Palavras-chave: Barras; Resfriamento; Transporte; Laminação.

\section{COOLING BARS SIMULATION BETEWEEN HEATING FURNACE AND ROLING MILL}

\section{Abstract}

The temperature decrease between the exit of the furnace and the rolling mill entrance is determinant factor in the rolling process and the quality tube. This study aims to model the cooling of the bars during transport in order to evaluate the minimum temperature required for the bar leaves the furnace and also the maximum transport time without compromising the rolling process, Allowing a better setup of equipment and reducing the production costs. The results obtained with the cooling model were compared with real values of temperature measurements by thermocouples installed on the bar and pyrometer measurements. Measurements with thermocouples were conducted in bars of 270,230 and $180 \mathrm{~mm}$ diameter. The pyrometers measurements were conducted in bars of $180 \mathrm{~mm}$ diameter. The error relative to the real values for all cases was below than $3 \%$.

Keywords: Cooling; Transport; Rolling Mill.

1 Estudante de engenharia mecânica, estagiário, Área de Tecnologia Térmica/Superintendência Geral de Produção, Vallourec Tubos do Brasil S.A., Belo Horizonte, Minas Gerais, Brasil

2 Engenheira Mecânica, Doutora, Engenheira de Processos Sênior, Área de Tecnologia Térmica/Superintendência Geral de Produção, Vallourec Tubos do Brasil S.A., Belo Horizonte, Minas Gerais, Brasil.

3 Engenheiro Mecânico, Doutor, Engenheiro de Processos Sênior, Área de Tecnologia Térmica/Superintendência Geral de Produção, Vallourec Tubos do Brasil S.A., Belo Horizonte, Minas Gerais, Brasil.

4 Estudante de engenharia mecânica, estagiário, Área de Tecnologia Térmica/Superintendência Geral de Produção, Vallourec Tubos do Brasil S.A., Belo Horizonte, Minas Gerais, Brasil. 


\section{INTRODUÇÃO}

O processo de produção de tubos sem costura tem como primeira etapa o aquecimento de barras metálicas a uma temperatura em torno de $1200^{\circ} \mathrm{C}$, após 0 aquecimento essas barras são transportadas até o laminador. A temperatura que a barra chega ao laminador é de grande importância para a qualidade do processo. Desta forma, visando entender e prever o processo de resfriamento das barras durante o transporte das mesmas foi desenvolvida uma ferramenta para simular o resfriamento das barras durante o transporte.

O modelo matemático desenvolvido por SILVA [1] e adaptado por Cançado [2] foi modificado para as condições de contorno deste problema, desse modo foi possível simular o perfil de resfriamento de uma barra durante o transporte entre o forno de aquecimento e o laminador.

O conhecimento do perfil de resfriamento da barra permite determinar a mínima temperatura de desenfornamento e o tempo maximo que a barra pode ficar fora do forno sem prejudicar o processo de laminação, possibilita também um setup mais eficiente dos equipamentos gerando redução nos custos de produção. Para construção do perfil de resfriamento foi realizado um balanço energético sobre a barra. O resultado do modelo foi validado com medições realizadas com termopares fixados na barra e também com pirômetro. Os resultados foram comparados para determinação da precisão do modelo.

\section{MATERIAIS E MÉTODOS}

\subsection{Modelo Matemático}

No modelo matemático de resfriamento, a barra foi dividida em $\mathrm{n}$ camadas. $\mathrm{A}$ camada superficial (camada 1) troca calor por radiação e convecção natural com o ambiente externo e por condução com a camada 2. As camadas internas (2 a n-1) trocam calor por condução. A camada interna $n$ tem área muito pequena, próxima de zero, portanto considera-se que a temperatura desta é igual a da camada $n-1$. As (figuras de 1 a 2 ) ilustram essa modelagem:

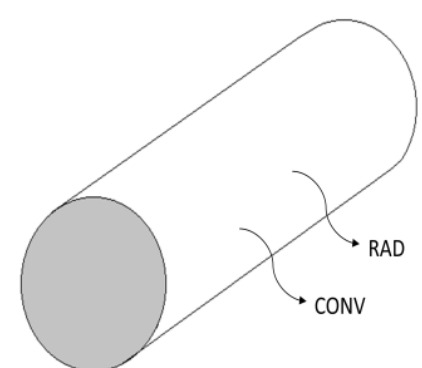

Figura 1. Transferência de Calor camada superficial

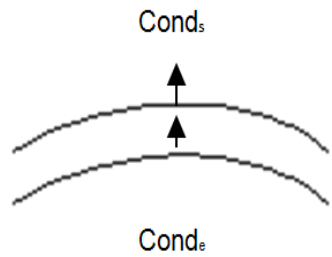

Figura 2. Transferência de Calor camada 2 a n-1 


\subsection{Balanço de energia}

O balanço de energia foi resolvido de maneira discreta, aproximando-se $d T / d t$ por $\Delta T / \Delta t[3]$. Foi adotado um passo de tempo, $\Delta t$, de 1 segundo e as propriedades dependentes da temperatura foram atualizadas a cada iteração. O equacionamento do problema é dado pelas (Equações de 1 a 3):

\section{Camada 1}

$$
m \cdot C_{p} \cdot \frac{\Delta T}{\Delta t}=h_{c o n v} \cdot A \cdot\left(T-T_{\infty}\right)+F \cdot h_{r a d} \cdot A \cdot\left(T-T_{\infty}\right)+k \cdot \frac{2 \pi}{L n \frac{r e}{r i}} \cdot\left(T_{1}-T_{2}\right)
$$

\section{Camada 2 a camada $n-1$}

$$
\text { m. } C_{p} \cdot \frac{\Delta T}{\Delta t}=k \cdot \frac{2 \pi}{L n \frac{r e}{r i}} \cdot\left(T_{n}-T_{n+1}\right)
$$

\section{Camada $\mathbf{n}$}

$$
T_{n}=T_{n-1}
$$

Em que: $m$ é a massa de uma barra, $C_{\mathrm{p}}$ é o calor específico do aço, $T$ é a temperatura da barra, $t$ é o tempo, $h_{\text {conv }}$ é o coeficiente de transferência de calor convectivo médio, $A$ é a área da barra, $F$ é o fator de forma, $h_{\text {rad }}$ é o coeficiente de transferência de calor por radiação. O coeficiente de transferência de calor por radiação foi definido pela (Equação 4):

$$
h_{\text {rad }}=\varepsilon \cdot \sigma \cdot\left(T+T_{\infty}\right)+\left(T^{2}+T_{\infty}{ }^{2}\right)
$$

Em que: $\varepsilon$ é a emissividade da superfície das barras de aço (igual a 0,91), $\sigma$ é a constante de Stefan Boltzmann cujo valor é $5,67 \cdot 10^{-8} \frac{\mathrm{W}}{\mathrm{m}^{2} \cdot \mathrm{K}^{4}}$. O coeficiente de transferência de calor convectivo foi definido pela (Equação 5):

$$
h_{\text {conv }}=\frac{\overline{N U} \cdot K}{D}
$$

Em que: $\overline{\mathrm{NU}} \equiv$ é o valor médio do número de Nusselt da barra, $k$ é a condutividade térmica, $D$ é o diâmetro da barra.

\subsection{Métodos numéricos}

Para resolução dos balanços de energia utilizou-se a técnica das substituições sucessivas ${ }^{[4]}$, onde a Temperatura é calculada como uma função da temperatura, como na (equação 6):

$$
T=f_{(T)}(6)
$$

O critério de convergência do método é dado pela (equação 7):

Onde: $E=0,001$

$$
\left|\frac{T_{k}-T_{k-1}}{T_{k}}\right| \leq E(7)
$$




\subsection{Medições experimentais}

As campanhas de medições experimentais foram realizadas em barras de 270,230 e $180 \mathrm{~mm}$ de diâmetro. Realizaram-se dois tipos de medidas:

1. Medições com termopares em barras de 270, 230 e $180 \mathrm{~mm}$.

2. Medições com pirômetro em barras de $180 \mathrm{~mm}$.

\subsubsection{Medições com termopares}

Para medições com termopares, a barra é perfurada e termopares são fixados em diversas posições ao longo do comprimento, como na (figura 3):

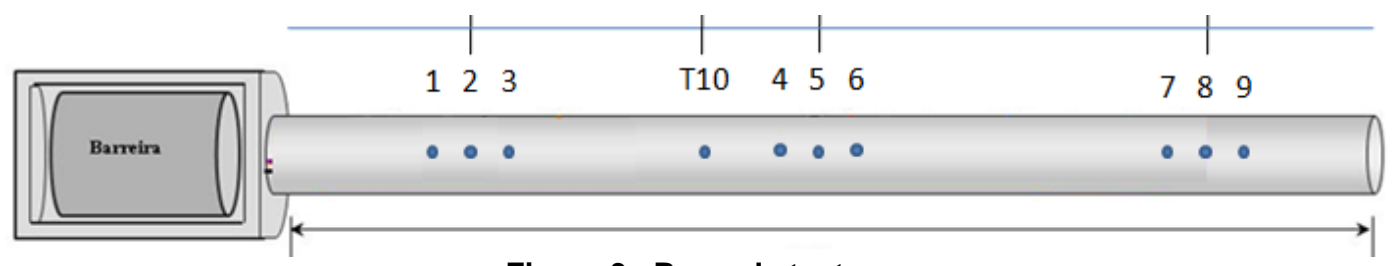

Figura 3. Barra de testes

Os termopares de numero 1, 4, 7 foram fixados a uma profundidade de $10 \mathrm{~mm}$ da face superior da barra. Os de numero 2, 5 e 8 a uma profundidade que corresponde ao centro da barra. Os de número 3, 6, 9 a uma profundidade de $10 \mathrm{~mm}$ da face inferior da barra. O termopar de numero 10 funcionou como "antena" medindo a temperatura na atmosfera do forno. Os termopares foram conectados a um logger que coleta e armazena informações a cada 5 segundos. Este logger foi alocado dentro de uma barreira, que tem como finalidade protegê-lo da alta temperatura na atmosfera do forno. A barra de testes foi enfornada com as demais, registrando assim todo o processo de aquecimento e também o de resfriamento após esta ser desenfornada. Os dados do logger foram então descarregados, e foneceram o perfil de aquecimento e resfriamento da barra. Desta forma, este ensaio permitiu a comparação entre o perfil simulado e o perfil calculado pelo modelo matemático.

\subsubsection{Medições com pirômetro}

As medições com pirômetro consistiram na coleta de valores de temperatura das barras em dois momentos, na saída do forno e na entrada do laminador. O tempo de transporte foi cronometrado. Assim, foi possível a comparação entre a temperatura real da barra e a temperatura calculada para um determinado tempo de transporte.

\section{RESULTADOS E DISCUSSÃO}

\subsection{Medições com Termopar}

O perfil de temperatura das barras durante o resfriamento foi simulado e pode ser comparado ao perfil medido com os termopares. Foi utilizada para análise a temperatura simulada para a camada 2 da barra, que corresponde a profundidade na qual o termopar foi fixado. O valor inicial de temperatura utilizado na simulação corresponde ao valor real das medições com termopar no inicio do resfriamento. $O$ perfil de resfriamento para os três diâmetros medidos pode ser observados nas figuras abaixo: 


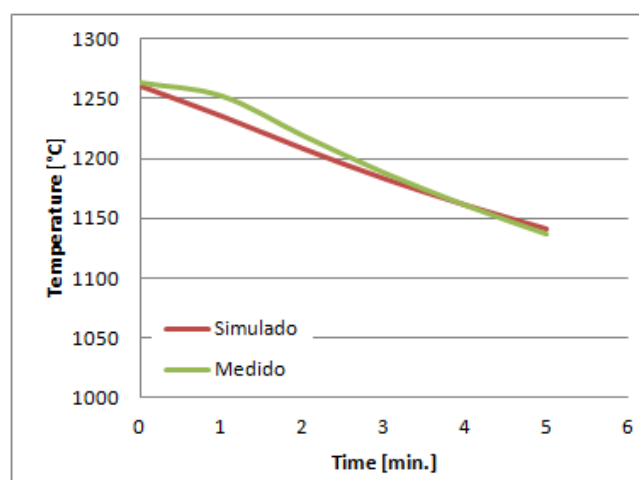

Figura 4. Perfil de Resfriamento da barra de $270 \mathrm{~mm}$

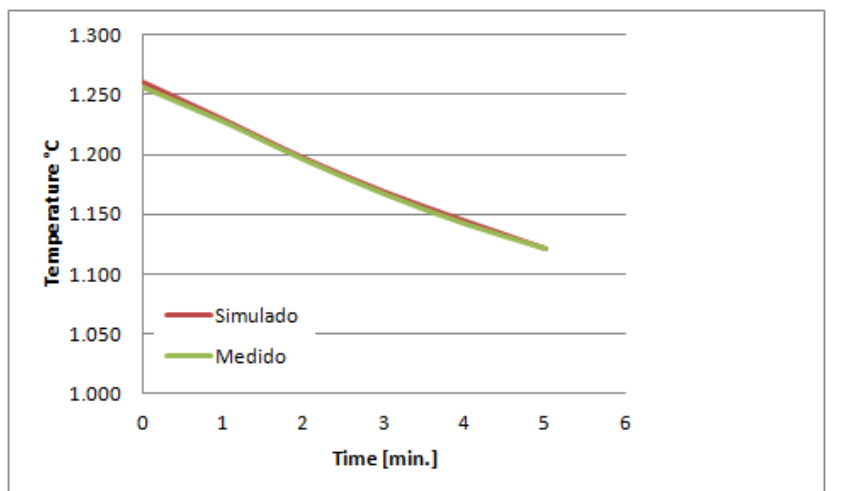

Figura 5. Perfil de Resfriamento barra de $230 \mathrm{~mm}$

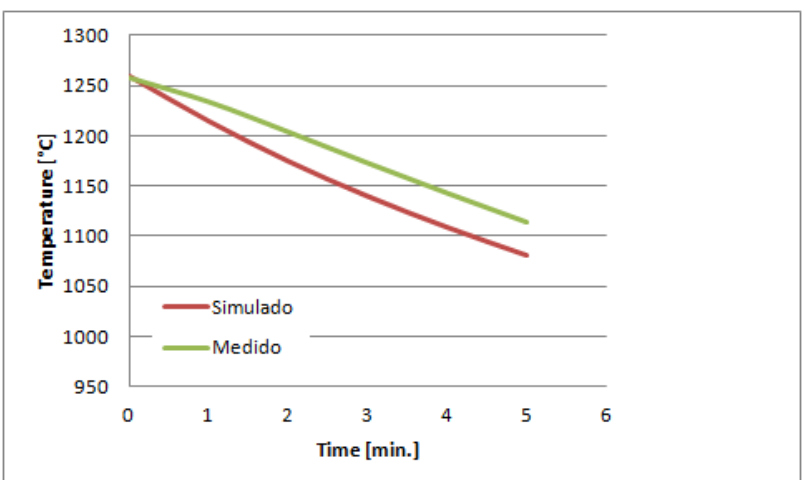

Figura 6. Perfil de Resfriamento da barra de $180 \mathrm{~mm}$

Os valores de temperatura em relação ao tempo da simulação das medições podem ser visto nas (tabelas de 1 a 3 ):

Tabela 1. Valores de temperatura simulados medidos para barras de $180 \mathrm{~mm}$

$0 \mathrm{~min} .1 \mathrm{~min}$. $2 \mathrm{~min}$. $3 \mathrm{~min} .4 \mathrm{~min}$. $5 \mathrm{~min}$.

\begin{tabular}{lllllll}
\hline Simulado $\left[{ }^{\circ} \mathrm{C}\right]$ & 1263 & 1235 & 1208 & 1183 & 1161 & 1141 \\
\hline Medido $\left[{ }^{\circ} \mathrm{C}\right]$ & 1263 & 1252 & 1219 & 1188 & 1161 & 1137 \\
\hline
\end{tabular}

O Temperatura simulada da camada 2 que tem profundidade próxima a do termopar 4 A temperatura no tempo zero corresponde a valor real medido no inicio do resfriamento

Tabela 2. Valores de temperatura simulados medidos para barras de $230 \mathrm{~mm}$ $0 \mathrm{~min} .1 \mathrm{~min}$. $2 \mathrm{~min}$. $3 \mathrm{~min}$. $4 \mathrm{~min}$. $5 \mathrm{~min}$.

\begin{tabular}{lllllll}
\hline Simulado $\left[{ }^{\circ} \mathrm{C}\right]$ & 1257 & 1229 & 1197 & 1169 & 1145 & 1122 \\
\hline Medido $\left[{ }^{\circ} \mathrm{C}\right]$ & 1257 & 1228 & 1196 & 1167 & 1142 & 1121 \\
\hline
\end{tabular}

O Temperatura simulada da camada 2 que tem profundidade próxima a do termopar 4 A temperatura no tempo zero corresponde a valor real medido no inicio do resfriamento 
Tabela 3. Valores de temperatura simulados medidos para barras de $180 \mathrm{~mm}$

\begin{tabular}{lcccccc}
\hline & $\mathbf{0}$ min. & $\mathbf{1}$ min. & $\mathbf{2}$ min. & $\mathbf{3}$ min. & $\mathbf{4}$ min. & $\mathbf{5}$ min. \\
\hline Simulado $\left[{ }^{\circ} \mathrm{C}\right]$ & 1258 & 1215 & 1175 & 1140 & 1109 & 1081 \\
\hline Medido $\left[{ }^{\circ} \mathrm{C}\right]$ & 1258 & 1234 & 1204 & 1173 & 1143 & 1114 \\
\hline
\end{tabular}

O Temperatura simulada da camada 2 que tem profundidade próxima a do termopar 4

A temperatura no tempo zero corresponde a valor real medido no inicio do resfriamento

O erro entre a simulação e os dados experimentais foi calculado de acordo com a (Equação 8):

$$
\text { Erro }=\frac{\text { Valor medido-Valor simulado }}{\text { Valor medido }} * \mathbf{1 0 0}
$$

(A Tabela 4) apresenta os valores de erro calculados, para as barras de cada um dos três diâmetros analisados, em função do tempo.

Tabela 4. Erro do modelo matemático em relação às medidas realizadas com termopar

\begin{tabular}{lrrrrrr}
\hline & 0 min. & 1 min. & 2 min. & 3 min. & 4 min. & 5 min. \\
\hline $270 \mathrm{~mm}$ & - & $1,36 \%$ & $0,90 \%$ & $0,42 \%$ & $0,00 \%$ & $0,35 \%$ \\
\hline $230 \mathrm{~mm}$ & - & $0,08 \%$ & $0,08 \%$ & $0,17 \%$ & $0,26 \%$ & $0,09 \%$ \\
\hline $180 \mathrm{~mm}$ & - & $1,54 \%$ & $2,41 \%$ & $2,81 \%$ & $2,97 \%$ & $2,96 \%$ \\
\hline
\end{tabular}

(A Tabela 5) apresenta os valores de erro médio calculado para as barras de cada um dos três diâmetros analisados.

Tabela 5. Erro Médio em relação às medidas realizadas com termopar

\begin{tabular}{ll}
\hline $270 \mathrm{~mm}$ & $0,51 \%$ \\
\hline $230 \mathrm{~mm}$ & $0,11 \%$ \\
\hline $180 \mathrm{~mm}$ & $2,12 \%$ \\
\hline
\end{tabular}

\subsection{Medições com Pirômetro}

A temperatura inicial da simulação é a temperatura media de desenfornamento da barra (valor medido), A temperatura final da simulação corresponde a temperatura na entrada do laminador. Os resultados obtidos com a simulação do resfriamento das barras e os valores coletados nas campanhas de medições realizadas com 0 pirômetro são apresentados na (Tabela 6).

Tabela 6. Valores de temperatura simulados e medidos para barras de $180 \mathrm{~mm}$

\begin{tabular}{lcc}
\hline & Inicio & Fim \\
\hline Simulado $\left[{ }^{\circ} \mathrm{C}\right]$ & 1110 & 1077 \\
\hline Medido $\left[{ }^{\circ} \mathrm{C}\right]$ & 1110 & 1073 \\
\hline
\end{tabular}

A temperatura simulada corresponde a camada superficial equivalente a medida pelo pirômetro.

Segundo a (Equação 6) o erro dos valores de temperatura simulados em relação aos medidos com pirômetro é de $\mathbf{0 , 3 7} \%$. 


\section{CONCLUSÃO}

O modelo desenvolvido é capaz de simular o perfil de resfriamento das barras no transporte entre a saída do forno a entrada do laminador. O entendimento deste resfriamento permite o correto setup dos equipamentos, melhorando a qualidade dos tubos laminados e reduzindo os custos com:

- Retrabalho;

- Energia no aquecimento desnecessário das barras;

- Energia na laminação de barras frias;

- Desgaste prematuro das ferramentas;

De maneira geral, o modelo de resfriamento apresentou boa precisão obtendo erro médio inferior a 2,5\%. Desta forma, alcançou de maneira satisfatória seu objetivo, auxiliar a tomada de decisões no ambiente industrial.

Para as medições com termopares a simulação do resfriamento das barras de 270 e $230 \mathrm{~mm}$ apresentou erro realtivo inferior $0,5 \%$ indicando uma boa precisão do modelo. Para barras de $180 \mathrm{~mm}$ a simulação apresentou erro realtivo de aproximadamente $2 \%$, sendo este, o pior resultado dentre os três tipos de barras estudados. Vale ressaltar que o processo de aquecimento e de transporte, neste caso, ocorreu em equipamentos diferentes das barras de 270 e $230 \mathrm{~mm}$ (linhas de produção diferentes). Assim, a configuração deste equipamento pode influenciar a precisão do modelo. Faz-se necessário um estudo mais detalhado para refinar o modelo neste caso.

As medidas de temperatura coletadas com pirômetro apresentaram um erro relativo de aproximadamente $0,3 \%$ em relação à temperatura simulada. Este resultado difere significativamente dos resultados obtidos com as medições por termopares (para blocos de $180 \mathrm{~mm}$ ). É importante destacar, que fatores como formação de carepa e distância entre o pirômetro e a barra aumentam o grau de incerteza das medições realizadas com pirômetro. Tais fatores explicam a diferença de precisão do modelo para as medições realizadas com pirômetro e termopares.

\section{Agradecimentos}

À empresa Vallourec Tubos do Brasil S.A. por fomentar trabalhos de pesquisa e desenvolvimento.

\section{REFERÊNCIAS}

1 SILVA, P. C. S., "Analise numérica e experimental do resfriamento do carregamento de barras de aço." 2014. 82f. Dissertação (Mestrado) - Universidade Federal de Minas Gerais, Programa de Pós-Graduação em engenharia mecânica, Belo Horizonte, 2014.

2 Cançado, K. N, Soares, L. N, Silva, J. R., Rodrigues, L. F., Modelo Matemático Para o Calculo do Resfriamento de barras em bolsas de aciaria. $37^{\circ}$ Seminário de balanços Energéticos Globais e Utilidades. (No Prelo)

3 INCROPERA, F. P., DeWITT, D. P., Fundamentos de transferência de calor e de massa, 5aed. Rio de Janeiro: LTC, 2003, 698p.

4 CHAPRA, S. C., Applied Numerical Methods With MATLAB, 3aed. Rio de Janeiro: MC Graw Hill, 2004, 384p.. 